Original Article

\title{
Gait changes after using a temporomandibular joint exerciser in patients who underwent lower limb joint surgery
}

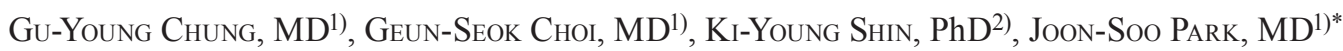 \\ 1) Burteam Hospital: Osan-dong, Osan City, Gyeonggi-do, Republic of Korea \\ 2) Department of Energy and Power Conversion Engineering, University of Science and Technology, \\ Republic of Korea
}

\begin{abstract}
Purpose] The improvements in gait of the patients with lower limb disease who used a temporomandibular joint (TMJ) exerciser were verified. [Subjects and Methods] Eleven subjects were included. Their mean age was 53.2 years. The lower limb joint angles before and after using the TMJ exerciser were measured using a gait analyzer. Before the gait experiment, the TMJ exerciser setting process and one-leg stance balance test (OLST) were repeated until the balance maintenance time improved. [Results] Because of the OLST, the mean change in the body center point after the subjects used the exerciser improved from $5.76 \mathrm{~mm}$ to $4.20 \mathrm{~mm}$. When the TMJ exerciser was used, the joint angle range of the subjects approached that of the normal individuals. [Conclusion] According to the gait experiments, the angles of the subjects' hips, knees, and ankle joints approached to those of the normal individuals after the subjects used the TMJ exerciser; however, the results did not completely match. The changes in the hip, knee, and ankle joint angles were statistically significant, which confirm the usefulness of the TMJ exerciser.

Key words: Temporomandibular joint, Gait, Body balance
\end{abstract}

(This article was submitted Dec. 15, 2015, and was accepted Feb. 3, 2016)

\section{INTRODUCTION}

Abnormal loads on the lower limb joints induced by sedentary lifestyles, crossed legs, or high-heel shoes usage, or by congenital or acquired factors such as curbed legs and car accidents can cause a medial/lateral muscle imbalance around the knee joint ${ }^{1-3)}$. Consequently, the patella moves abnormally when the knee is flexed or extended, and symptoms such as anterior knee pain (AKP) ${ }^{4}$ and changes in gait develop because of pain in the lower limb joints ${ }^{5-7)}$. These changes can cause abnormal gaits, excessive loads on specific joints, or muscle fatigue on specific muscles ${ }^{8)}$. The methods of changing gait include surgical procedures ${ }^{9)}$, rehabilitation ${ }^{10,11)}$, and combinations of the two ${ }^{12}$. Rehabilitation takes a longer time compared to surgical procedures; however, it helps the damaged nerves, muscles, and joints to recover to the normal or close to the normal state ${ }^{13}$.

Rehabilitation therapy includes physical therapy, therapeutic exercise, manual therapy, and treatments using instruments ${ }^{14)}$. Since therapeutic exercise and manual therapy require physical therapists, the patients should visit a medical facility. In comparison, the treatments using props and instruments can be received at any time and at any place by the patient themselves ${ }^{15}$ ). In direct rehabilitation, joint rehabilitation exercisers, osteopathic exercisers ${ }^{16)}$, and exoskeleton robots ${ }^{17)}$ are used to induce changes in gait and balance by applying forces to the lower limbs. In indirect rehabilitation, in contrast, instruments are applied to the areas other than the lower limbs to induce changes in gait and balance. This method does not produce any resistance from the lower limbs, and the patient constraints are minimal.

\footnotetext{
*Corresponding author. Joon-Soo Park (E-mail: jsparkme@korea.ac.kr)

(C)2016 The Society of Physical Therapy Science. Published by IPEC Inc.

This is an open-access article distributed under the terms of the Creative Commons Attribution Non-Commercial No Derivatives (by-nc-nd) License $<$ http://creativecommons.org/licenses/by-nc-nd/4.0/>.
} 
The areas in which the indirect method for changing the gait can be used including the temporomandibular joint (TMJ). TMJ is a synovial joint positioned between the mandibular joint process and the cranium temporal bone. Any movement in the TMJ affects the other joints because of its compound structure ${ }^{18)}$; Owing to its structure and anatomical features, the TMJ interacts with the lumbar muscles that support the spine; hence, it affects the body balance and gait ${ }^{19,20)}$.

In this study, the usefulness of a TMJ exerciser in changing the gait of patients was verified.

\section{SUBJECTS AND METHODS}

Eleven patients who underwent knee or ankle joint surgery at the Rehabilitation Exercise Center of Burteam Hospital participated in this study. Their mean age, height, and body weight were 53.2 years, $154.8 \mathrm{~cm}$, and $59.3 \mathrm{~kg}$, respectively. To apply the exerciser onto the subjects, the one-leg stance balance test (OLST) was conducted before the subjects used the TMJ exerciser (NO SICK, Gibunjounsesang, South Korea) as shown in Fig. 1. Subsequently, the TMJ exerciser was worn and adjusted by repeated OLSTs. This process was repeated twice or thrice, until a stable OLST result was obtained. All the subjects understood the purpose of this study and agreed to participate. An informed consent form approved by the Institutional Review Board was signed by all the participants.

To measure the balance maintenance levels through OLST, an optical motion capture system (Optitrack, Natural Point, Ltd., USA) was used. Four markers were attached to the LASI, RASI, LPSI, and RPSI in the Helen Hayes marker set. The unipedal stance test was conducted with the eyes closed and the arms crossed, until the subjects could no longer maintain their balance. The test was repeated four times before and after applying the TMJ exerciser. To measure the stability, the pelvic center, the center point of the four markers in the unipedal stance test, was calculated. The anterior/posterior, medial/ lateral, and vertical movements of the center point were measured.

To investigate the change before and after using the exerciser, optical motion capture system (Optitrack, Natural Point, Ltd., USA) was used. Sixteen markers (one each) were attached for gait measurement at the LASI, RASI, LPSI, RPSI, RTHI, LTHI, RKNE, LKNE, RTIB, LTIB, RANK, LANK, RTOE, LTOE, RHEE, and LHEE.

To ensure a natural gait that is free from any restraint, each subject was asked to walk a 5-m path thrice with and without the exerciser. By using the obtained marker data, the flection/extension angles of the hips, knees, and ankle joints were calculated during one gait cycle that started from the initial contact of the observed limb to the next contact. The calculated angles of the subjects were compared with those of the non-disabled individuals.

Statistical analyses were performed by using SPSS version 14 for Windows (SPSS Institute Korea, Seoul, South Korea), and the results were presented as mean \pm standard deviation (SD). To analyze the changes in gait before and after the test, paired t-test was performed to measure the changes within the group. Statistical significance was accepted for $\mathrm{p}<0.05$.

\section{RESULTS}

Because the subjects were in the age of $60 \mathrm{~s}$ and $70 \mathrm{~s}$, the average balance maintenance time was measured between 3 and 4 seconds in OLST ${ }^{21)}$. The pelvic center point was calculated using the four markers attached to the pelvic area, and the means and SDs of the pelvic center point movements were additionally calculated. As shown in Table 1, mean values of $5.76 \mathrm{~mm}$ in the anterior/posterior, $4.16 \mathrm{~mm}$ in the medial/lateral, and $1.89 \mathrm{~mm}$ in the vertical movements were obtained. After the subjects applied the exerciser, mean values of $4.20 \mathrm{~mm}$ in the anterior/posterior, $2.69 \mathrm{~mm}$ in the medial/lateral, and $1.55 \mathrm{~mm}$ in the vertical movements were obtained. The reason for lower SD measured in all the directions after using the TMJ exerciser was less shaking during the unipedal test, which meant less energy expended for maintaining balance.

For an easy comparison, the range of joint angles from the normal individuals and the subjects was plotted on the graphs with two conditions before and after using the TMJ exerciser.

The hip joint angle changes among the gaits are shown in Fig. 2(a). Although the hip joint angle results before and after using the TMJ exerciser were not within the normal range, the angle approached the normal range in 40-60\% region of the gait cycle after the subjects used the TMJ exerciser. The change between before and after using the TMJ exerciser was $\mathrm{p}<0.001$, which confirmed a statistically significant difference. The change in knee joint angle among gaits is shown in Fig. 2(b). The change between before and after using the TMJ exerciser was significant at $0-60 \%$ angle range, which corresponds to the stance phase. The angle change before and after using the TMJ exerciser was $p<0.001$, which confirmed a statistically significant difference. The ankle joint angle change among gaits is shown in Fig. 2(c). The gait of subjects was different from that of the normal individuals in all domains; however, the angle change before and after using the TMJ exerciser was $\mathrm{p}<0.001$, which confirmed a statistically significant difference. Although the use of the TMJ exerciser could not change the subjects' gaits to make it within the normal range, the graph pattern approached the normal range after the subjects used the TMJ exerciser, particularly during the stance phase where the feet were fixed on the floor. Considering that the stance phase is the moment when the body weight is applied to each joint, improvements in the joint angle during the stance phase are expected to reduce the pain during the rehabilitation period. 


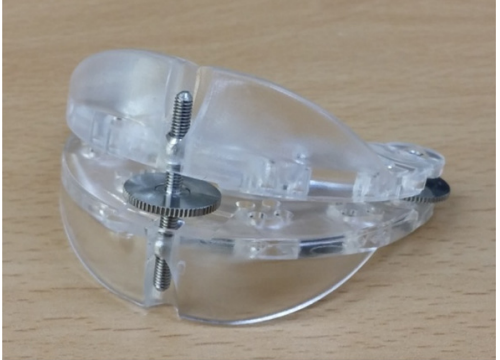

Fig. 1. TMJ exerciser

Table 1. The mean and standard deviation of movement of pelvic center points (unit: $\mathrm{mm}$ )

\begin{tabular}{lcc}
\hline & Before & After \\
\hline Sagittal & $5.76 \pm 2.46$ & $4.20 \pm 2.15$ \\
Coronal & $4.16 \pm 2.96$ & $2.69 \pm 1.46$ \\
Axial & $1.89 \pm 0.92$ & $1.55 \pm 0.92$ \\
\hline
\end{tabular}
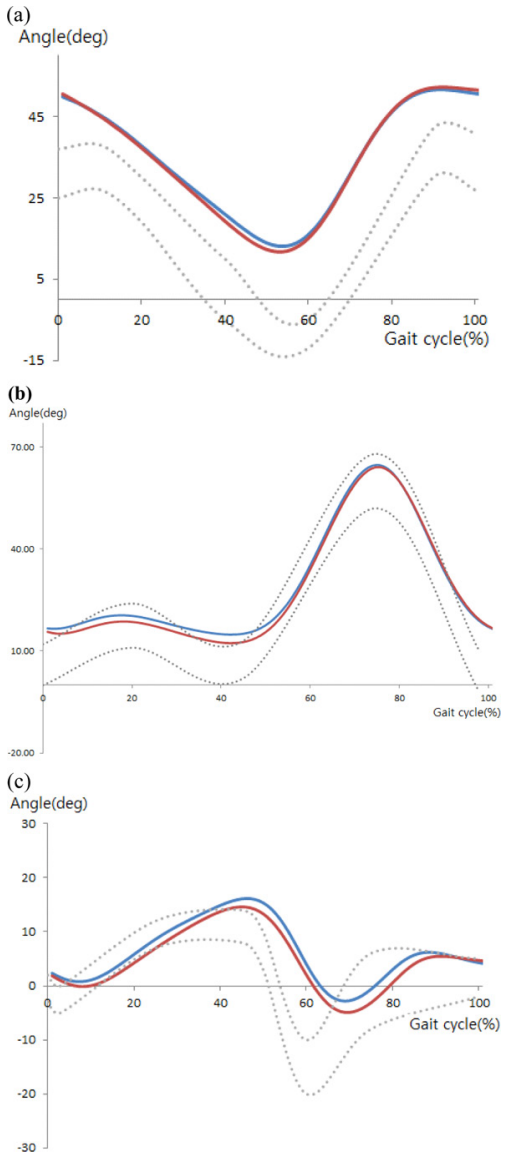

Fig. 2. Joint angle changes before and after using the TMJ exerciser

Blue line for before using the TMJ exerciser, red line for after using the TMJ exerciser, and dashed line for the range of normal gait. Hip joint (a); knee joint (b); and ankle joint (c)

\section{DISCUSSION}

In this study, a TMJ exerciser was developed with the purpose of rehabilitating patients who underwent knee or ankle surgery, and the effectiveness of the exerciser was verified through the OLST, TMJ change experiments, and gait tests. In OLST, the TMJ exerciser was set for each subject. Consequently, less shaking of the pelvic center point was observed during the unipedal stance test after the subjects applied the TMJ exerciser because of the increased stability. In the test for measuring the changes in the facial symmetry after using the TMJ exerciser, the marker changes of the 11 subjects before and after using the TMJ exerciser were quantitatively measured considering the individual differences. The goal to improve the facial symmetry could be attained after using the TMJ exerciser. The facial symmetry did not improve in this study because the TMJ exerciser was adjusted during the OLST to maintain the body balance after surgery. Instead, not only the TMJ positions but also the facial symmetry was affected after the subjects used the TMJ exerciser ${ }^{22}$. Although the hip, knee, and ankle joint angles were not within the normal range, they approached the normal range after the subjects used the TMJ exerciser. In addition, statistically significant differences in the hip, knee, and ankle angles were observed, which confirmed the changes in each joint after the subjects used the exerciser. Particularly, the normalization tendency was significant during the stance phase, which induced gait pain. Accordingly, the pain during the rehabilitation period is expected to lessen.

The limitation of this study was the small sample size (11 subjects). In the future studies, radiography or imaging technologies may be used to measure directly the TMJ changes with a larger sample group. In addition, test methods accompanying additional treatment protocols need to be developed. 


\section{ACKNOWLEDGEMENT}

The authors express their appreciation for the time and efforts of Sungwan Lee, P.T., Manager of Rehabilitation Exercise Center, Burteam Hospital and his members for this study.

\section{REFERENCES}

1) Henrichs A: A review of knee dislocations. J Athl Train, 2004, 39: 365-369. [Medline]

2) Grace TG, Sweetser ER, Nelson MA, et al.: Isokinetic muscle imbalance and knee-joint injuries. A prospective blind study. J Bone Joint Surg Am, 1984, 66: 734-740. [Medline]

3) Kim MJ, Kim SY, Hwang KK, et al.: Ultrasonography analysis of knee range of motion and the quadriceps femoris of patients with knee osteoarthritis. J Phys Ther Sci, 2012, 24: 461-463. [CrossRef]

4) Cesarelli M, Bifulco P, Sansone M, et al.: Evaluation of quadriceps muscles in anterior knee pain: a possible sport medicine application. Biomech Sports, 2004, 473: 53-58.

5) Noyes FR, Dunworth LA, Andriacchi TP, et al.: Knee hyperextension gait abnormalities in unstable knees. Recognition and preoperative gait retraining. Am J Sports Med, 1996, 24: 35-45. [Medline] [CrossRef]

6) Coombs R, Garbutt G: Developments in the use of the hamstring/quadriceps ratio for the assessment of muscle balance. J Sports Sci Med, 2002, 1: 56-62. [Medline]

7) Lee A, Park J, Lee S: Gait analysis of elderly women after total knee arthroplasty. J Phys Ther Sci, 2015, 27: 591-595. [Medline] [CrossRef]

8) Kim HD: Muscle eccentric control in gait initiation. Phys Ther Korea, 2001, 8: 81-89.

9) Levinger P, Menz HB, Morrow AD, et al.: Knee biomechanics early after knee replacement surgery predict abnormal gait patterns 12 months postoperatively. J Orthop Res, 2012, 30: 371-376. [Medline] [CrossRef]

10) Dickstein R: Rehabilitation of gait speed after stroke: a critical review of intervention approaches. Neurorehabil Neural Repair, 2008, 22: 649-660. [Medline] [CrossRef]

11) Park BS, Kim MY, Lee LK, et al.: Effects of conventional overground gait training and a gait trainer with partial body weight support on spatiotemporal gait parameters of patients after stroke. J Phys Ther Sci, 2015, 27: 1603-1607. [Medline] [CrossRef]

12) The Korean Pain Society: Textbook of Pain Medicine, 4th ed. Shin-won Publishing, 2012.

13) Belda-Lois JM, Mena-del Horno S, Bermejo-Bosch I, et al.: Rehabilitation of gait after stroke: a review towards a top-down approach. J Neuroeng Rehabil, 2011, 8: 66-84. [Medline] [CrossRef]

14) Carolyn K, Lynn AC: Therapeutic Exercise: Foundations and Techniques, 6th ed. F.A. Davis, 2012.

15) Oh SH, Yoo KT: Effect of gym-ball exercise program to pelvis and spine of the 20's woman. J Kor Soc Phys Med, 2014, 9: 339-345. [CrossRef]

16) Mayr A, Kofler M, Quirbach E, et al.: Prospective, blinded, randomized crossover study of gait rehabilitation in stroke patients using the Lokomat gait orthosis Neurorehabil Neural Repair, 2007, 21: 307-314. [Medline] [CrossRef]

17) Ahamed NU, Sundaraj K, Ahmad RB, et al.: Recent survey of automated rehabilitation systems using EMG biosensors. J Phys Ther Sci, 2011, 23: 945-948. [CrossRef]

18) Perinetti G, Contardo L, Silvestrini-Biavati A, et al.: Dental malocclusion and body posture in young subjects: a multiple regression study. Clinics (Sao Paulo), 2010, 65: 689-695. [Medline] [CrossRef]

19) Saito ET, Akashi PM, Sacco IC: Global body posture evaluation in patients with temporomandibular joint disorder. Clinics (Sao Paulo), 2009, 64: 35-39. [Medline] [CrossRef]

20) Silva PF, Biasotto-Gonzalez DA, Motta LJ, et al.: Impact in oral health and the prevalence of temporomandibular disorder in individuals with Parkinson's disease. J Phys Ther Sci, 2015, 27: 887-891. [Medline] [CrossRef]

21) Springer BA, Marin R, Cyhan T, et al.: Normative values for the unipedal stance test with eyes open and closed. J Geriatr Phys Ther, 2007, 30: 8-15. [Medline] [CrossRef]

22) Yun HI, Park JS, Chung KY, et al.: Evaluation of orthodontics for treating temporo-mandibular joint disorders using a stereo camera. J Kor Insti Info, Electro Comm Tech, 2015, 8: 359-366. 